\title{
Dust evolution in protoplanetary disks
}

\section{Jean-François Gonzalez ${ }^{1}$, Laure Fouchet ${ }^{2}$, Sarah T. Maddison ${ }^{3}$ and Guillaume Laibe ${ }^{1}$}

\author{
${ }^{1}$ Université de Lyon, Lyon, F-69003, France; Université Lyon 1, Villeurbanne, F-69622, France; \\ CNRS, UMR 5574, Centre de Recherche Astrophysique de Lyon, \\ École Normale Supérieure de Lyon, 46 allée d'Italie, F-69364 Lyon cedex 07, France \\ email: Jean-Francois.Gonzalez@ens-lyon.fr, Guillaume.Laibe@ens-lyon.fr \\ ${ }^{2}$ Department of Physics, ETH Zurich, CH-8093 Zurich, Switzerland \\ email: fouchet@phys.ethz.ch \\ ${ }^{3}$ Centre for Astrophysics and Supercomputing, Swinburne University of Technology, \\ PO Box 218, Hawthorn, VIC 3122, Australia \\ email: smaddison@swin.edu.au
}

\begin{abstract}
We investigate the behaviour of dust in protoplanetary disks under the action of gas drag using our 3D, two-fluid (gas+dust) SPH code. We present the evolution of the dust spatial distribution in global simulations of planetless disks as well as of disks containing an already formed planet. The resulting dust structures vary strongly with particle size and planetary gaps are much sharper than in the gas phase, making them easier to detect with ALMA than anticipated. We also find that there is a range of masses where a planet can open a gap in the dust layer whereas it doesn't in the gas disk. Our dust distributions are fed to the radiative transfer code MCFOST to compute synthetic images, in order to derive constraints on the settling and growth of dust grains in observed disks.
\end{abstract}

Keywords. planetary systems: protoplanetary disks - hydrodynamics - methods: numerical

\section{Introduction}

Dust grains in disks around young stars are thought to be the building blocks of planets (Dominik et al. 2007). They grow via sticking in low-velocity collisions from (sub)micron sizes up to decimetric sizes. The higher collision velocities of larger grains makes them shatter upon impact and prevents them from growing further. Yet we know that they must reach planetesimal sizes. How they overcome the decimetric barrier is the subject of much debate. Part of the solution to this problem could be found in the reduction of collision velocities via the increase of the dust layer density. This can be achieved by the settling of dust to the disk midplane.

Dust is also essential to the interpretation of disk observations. Indeed, the dust opacity largely dominates that of the gas, except in particular lines of molecular gas, which are more difficult to detect than dust thermal emission. Therefore, most images of disks trace the spatial distribution of dust, not that of the gas. Both components are often assumed to be well mixed, with a uniform gas-to-dust mass ratio of 100, but, as discussed in Sect. 2, this is not always the case and one has to be careful when deriving gas distributions from observations of dust in circumstellar disks.

We present here our work on dust evolution in protoplanetary disks using 3D SPH simulations. We treat vertical settling and radial migration of dust in planetless disks in Sect. 2 and present the resulting synthetic images and comparison to observations in Sect. 3. We describe the variety of structures of disks with gap-forming planets in Sect. 4 and our first treatment of grain growth in Sect. 5 . 

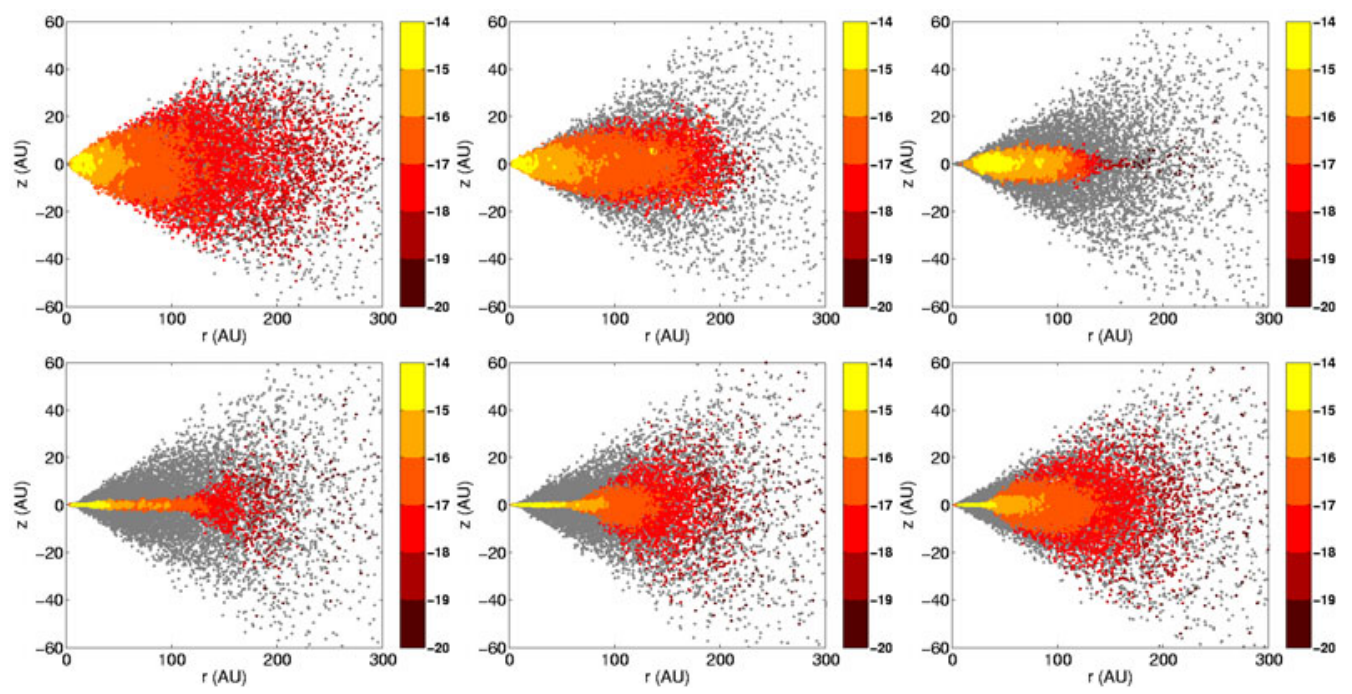

Figure 1. Gas (grey) and dust (colour represents $\log \rho_{\text {dust }}$, in $\mathrm{g} \mathrm{cm}^{-3}$ ) distributions in our CTTS disk viewed edge-on. Dust grain sizes are, from left to right and top to bottom: $10 \mu \mathrm{m}, 100 \mu \mathrm{m}$, $1 \mathrm{~mm}, 1 \mathrm{~cm}, 10 \mathrm{~cm}, 1 \mathrm{~m}$.

\section{Settling and migration}

In a non self-gravitating disk, gas feels the gravity of the central star and its own pressure gradient. As a result, it will orbit at sub-keplerian velocities. On the other hand, dust grains do not interact with each other and only feel the star's gravity: they have keplerian orbits. In a disk with both components, this velocity difference gives rise to aerodynamic drag, which slows down the dust and makes it migrate radially towards the central star. In the vertical direction, drag makes the dust settle towards the midplane.

Analytical studies of the radial migration of solid particles have been done by Weidenschilling (1977) and of their vertical settling by Garaud et al. (2004). In order to simultaneously study both effects, we developped a 3D, bi-fluid (gas+dust), SPH code to model vertically isothermal, non self-gravitating dusty disks (Barrière-Fouchet et al. 2005). The two inter-penetrating phases representing gas and dust interact via aerodynamic drag. Grain growth is not taken into account at this point (see Sect. 5).

We ran simulations for a $0.01 M_{\odot}$ disk in orbit around a $1 M_{\odot}$ star, representative of a classical T Tauri star (CTTS) disk, and composed of $99 \%$ gas and $1 \%$ dust in mass. We obtained the resulting dust spatial distributions for a set of grain sizes ranging from $1 \mu \mathrm{m}$ to $10 \mathrm{~m}$ (Barrière-Fouchet et al. 2005), a selection of which is illustrated in Fig. 1.

We found that for small sizes $(1-10 \mu \mathrm{m})$, with the strongest drag force, the dust is so strongly coupled to the gas that it follows its motion and both components are well mixed. On the other hand, the drag force for large grain sizes $(1-10 \mathrm{~m})$ is weak and the dust is almost insensitive to the gas, it occupies the whole disk again. It is for the median sizes $(100 \mu \mathrm{m}-10 \mathrm{~cm})$ that all the interesting dynamics happens and the gas drag has a strong influence on the resulting distributions, showing important vertical settling in the inner regions and depletion in the outer disk due to inwards radial migration, with varying strength depending on grain size.

We would like to point out that the efficiency of both processes depends not only on grain size but also on the nebula parameters, via the densities. Therefore, the size of the fastest inwards migrating grains is not universal. It is commonly thought to always be of the order of $1 \mathrm{~m}$, as found by Weidenschilling (1977) for the minimum mass solar nebula 


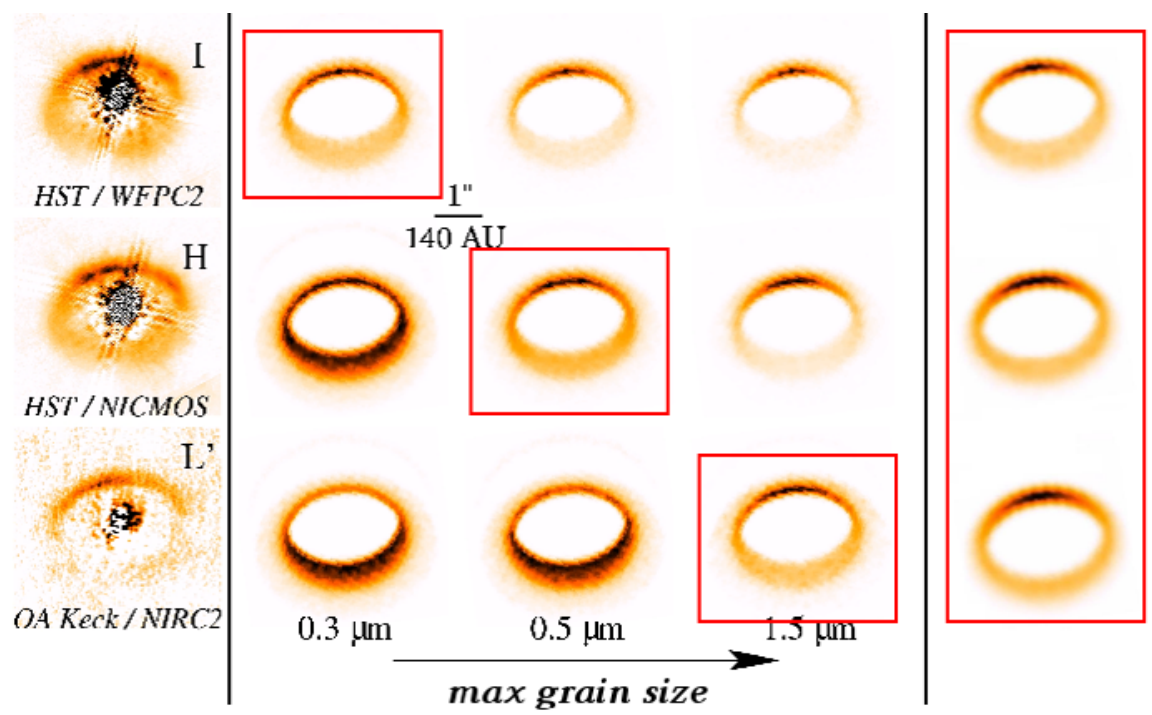

Figure 2. Observations (left panel) and synthetic images for models without (center panel) and with dust settling (right panel) of GG Tau's circumbinary ring in the I, H and L' bands.

(MMSN), whereas for our CTTS disk, we find it to be smaller, around $1 \mathrm{~mm}$ to $1 \mathrm{~cm}$, and to depend on the position in the disk.

\section{Synthetic images}

Numerous scattered light images of protoplanetary disks are now available (Watson et al. 2007). Light scattering depends on grain size and, because of the wavelength dependency of opacity, observations at different wavelengths reveal different depths in the disk. As a result, multi-wavelength observations in scattered light constitute a probe of dust settling.

In order to link our hydrodynamical simulations to observations of disks, we used our resulting dust distributions as an input to MCFOST, a continuum 3D Monte-Carlo radiative transfer code (Pinte et al. 2006). It can produce synthetic scattered light images, as well as spectral energy distributions (SEDs) and polarization maps. We found that dust settling and migration cause wavelength variations of the disk apparent size and of the dark lane width, in agreement with observations. They also affect SEDs, causing a change of slope between 10 and $200 \mu \mathrm{m}$. However, these effects are small, and a single observation would not give strong indications on the amount of settling. It is with a combination of multi-wavelength, multi-technique observations that one can hope to efficiently constrain the dust distribution.

This is beautifully illustrated in the case of GG Tau. The left panel of Fig. 2 shows three scattered light images of its circumbinary ring observed in the I, H, and L' bands. The middle panel displays the corresponding synthetic images for three models without dust settling, therefore with gas and dust well mixed in the disk, for which the dust size distribution (with the same slope as that of the interstellar medium), is truncated at a maximum size of $0.3,0.5$ and $1.5 \mu \mathrm{m}$, from left to right. No single model is able to simultaneously reproduce the observations in all three bands, and in particular the contrast between the front and back sides of the ring, but each one seems appropriate for a single band. This points towards a variation of the dust size distribution with depth in the disk. The right panel of Fig. 2 shows synthetic images produced from new 

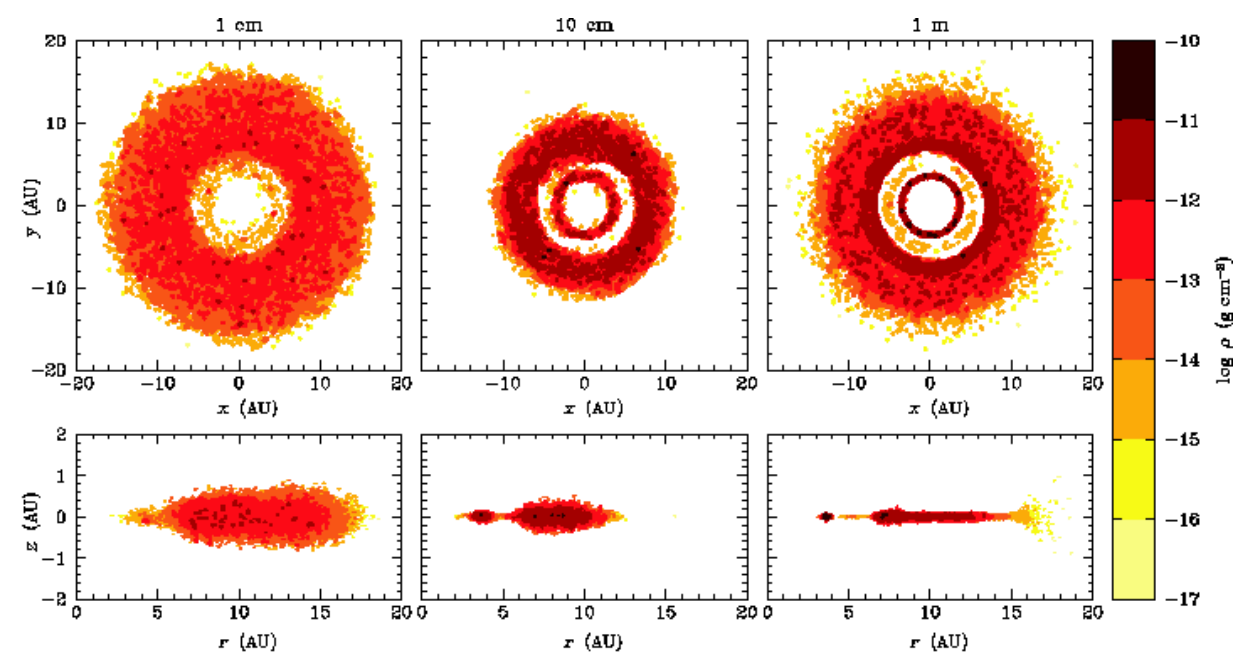

Figure 3. Gap created in the dust phase of the MMSN disk for 1-cm, 10-cm and 1-m grains, from left to right, by a $1 M_{\mathrm{J}}$ planet at $5.2 \mathrm{AU}$. Top panel: face-on view, bottom panel: edge-on view.

hydrodynamical simulations of GG Tau's circumbinary ring including dust settling and migration. Without any fit to the data, the agreement with observations is now much better, showing that dust had indeed settled in this ring. Polarization data comforting this result, as well as more detailed azimuthal brightness profile comparisons of observations and models, are presented in Pinte et al. (2007). The remaining discrepancies are likely due to missing physics in our simulations, among which grain growth.

\section{Planetary gaps}

In a disk containing a planet, gravitational perturbations of the planet cause density waves, leading to an exchange of angular momentum with the disk. The planet pushes the disk exterior (resp. interior) to its orbit further out (resp. in), therefore opening a gap. The gap is sustained if there is an equilibrium between tidal torques, acting to clear the gap, and viscous torques, acting to fill it. The observation of such a gap in a disk would provide a clear indication of the presence of a planet. Simulations of gaps in 2D gas disks by Wolf \& D'Angelo (2005) showed that a $1 M_{\mathrm{J}}$ planet can open a gap that will be detectable by ALMA at sub-mm wavelengths up to a distance of $100 \mathrm{pc}$. In 2D simulations of dusty disks, Paardekooper \& Mellema (2006) found that a smaller planet mass is needed to open a gap in the dust phase than in the gas.

We ran new 3D simulations of dusty disks with an embedded planet for two configurations, the CTTS disk we modeled previously and a MMSN disk, in which we vary the grain size and planet mass. We found that gap formation is much more rapid and striking in the dust layer rather than in the gas disk, and even more so than in 2D simulations (Maddison et al. 2007; Fouchet et al. 2007). Additionally, the structures caused in the dust phase depend strongly on the grain size, as illustrated in Fig. 3, due to varying drag and radial drift velocity throughout the disk.

Gaps are more prominent as the planet mass increases. Since they are also much deeper in the dust than in the gas, much lighter planets are needed to open one in the dust phase, the one visible in most disk observations, than in the gas phase. In our MMSN models, we found that a $0.2 M_{\mathrm{J}}$ planet can open a gap in the dust layer while only a small 

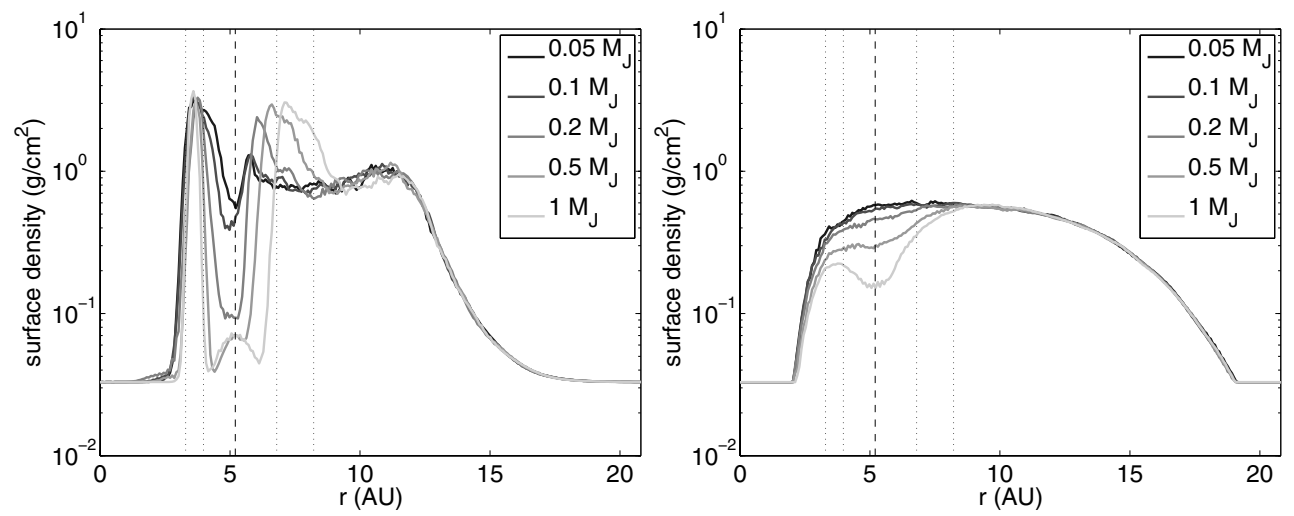

Figure 4. Azimuthally averaged surface density profile of the MMSN disk for the dust (1-m boulders, left) and the gas (right) with a planet of varying mass at $5.2 \mathrm{AU}$. The gas density is divided by 100 for direct comparison to the dust density.

surface density dip is observed in the gas (see Fig. 4). Lighter planets down to $0.05 M_{\mathrm{J}}$ create density dips in the dust, with steep gradients on both sides that should be visible by ALMA (Varnière et al. 2006). This suggests that planet gaps will be much easier to detect, with a lower threshold on planet mass, than previously anticipated.

Since the influences of grain size, affecting the disk thickness and radial profile (Fig. 3) and of planet mass, affecting the gap structure (Fig. 4) are different (see also Fouchet et al. 2007 for more detail), future observations of planet gaps will therefore allow to constrain the dust grain size distribution in addition to the planet mass.

Gaps can even play a role in planet formation. Because of a very efficient vertical settling, the volume density of the dust reaches that of the gas in several regions of the disk, and even exceeds it at the gap inner edge (see Fig. 5). This affects the dynamics in that region and can favour planetesimal growth, ultimately leading to the formation of a second planet in the vicinity of the first one.

\section{Grain growth}

We recently started to add grain growth in our code by implementing a scheme able to treat the variation of grain sizes via an analytical prescription. We tested it with the simple model of Stepinski \& Valageas (1997), who grow solid particles made of water ice by sticking, without taking shattering into account. We found that grain growth is very efficient, especially in the denser inner regions of the disk, but is too fast, as can be expected (Dullemond et al. 2005). For details, see the poster by Laibe (this volume) and references therein. More extensive tests are presented in Laibe et al. (2008).

\section{Conclusion}

Our results demonstrate that, with or without an embedded planet, gas and dust are not well mixed in protoplanetary disks. The density enhancements we find in the dust component shape their observed images, and help solid particles to aggregate.

Similarly to our work on GG Tau, we have started to compute synthetic images of disks with gaps in order to make better quantitative predictions of what ALMA will be able to detect. Gas dispersal, not taken into account here, can lower the drag force and alter the dust distribution, and should be considered when interpreting observations. 


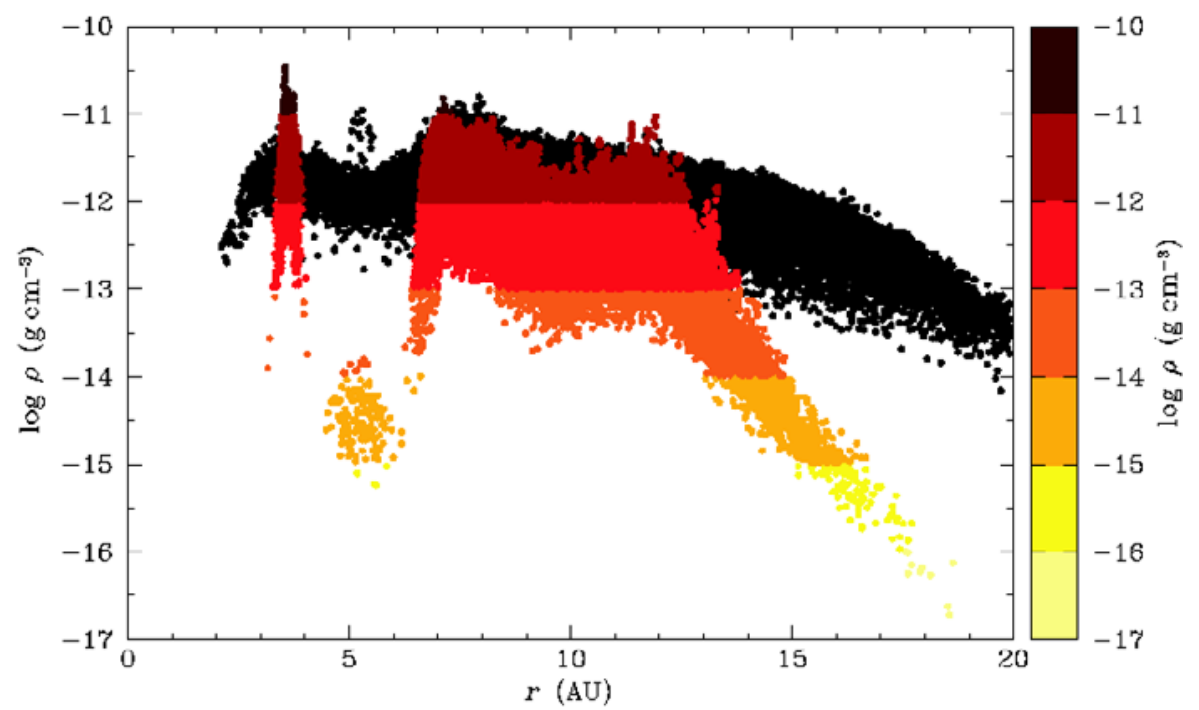

Figure 5. Volume density profile of the gas (black) and dust (colour) in the MMSN disk with a $1 M_{\mathrm{J}}$ planet at $5.2 \mathrm{AU}$ for 1 -m boulders.

Now that we have implemented in our code a mechanism to treat grain growth, we are working on an improved and more realistic physical model, taking into account detailed microscopic processes, as well as shattering, which produces small grains via collisional cascade. Our goal is to be able overcome the decimetric barrier. Our approach is different, but complementary to the one of Johansen et al. (2007), who find a concentration of solid particles in transient high pressure regions.

A physically consistent model of grain growth needs a proper implementation of turbulence in the gas, because it determines the kinematics of grains. As it is unfeasible to include a full treatment of turbulence in our code, we started working on a simplified, but realistic description.

\section{References}

Barrière-Fouchet, L., Gonzalez, J.-F., Murray, J. R., Humble, R. J., \& Maddison, S. T. 2005, $A \mathscr{E} A, 443,185$

Dominik, C., Blum, J., Cuzzi, J. N., \& Wurm, G. 2007, in: B. Reipurth, D. Jewitt \& K. Keil (eds.), Protostars and Planets $V$ (Tucson: University of Arizona Press), p. 783

Dullemond, C. P. \& Dominik, C. 2005, A\&A, 434, 971

Fouchet, L., Maddison, S. T., Gonzalez, J.-F., \& Murray, J. R. 2007, A\&A, 474, 1037

Garaud, P., Barrière-Fouchet, L., \& Lin, D. N. C. 2004, ApJ, 603, 292

Johansen, A., Oishi, J. S., Mac Low, M.-M., et al. 2007, Nature, 448, 1022

Laibe, G., Gonzalez, J.-F., Fouchet, L., \& Maddison, S. T. 2008, in preparation

Maddison, S. T., Fouchet, L., \& Gonzalez, J.-F. 2007, ApESSS, 311, 3

Paardekooper, S.-J. \& Mellema, G. 2006, A\&A, 453, 1129

Pinte, C., Ménard, F., Duchêne, G., \& Bastien, P. 2006, A\&A, 459, 797

Pinte, C., Fouchet, L., Ménard, F., Gonzalez, J.-F., \& Duchêne, G. 2007, A\&AA, 469, 963

Stepinski, T. F. \& Valageas, P. 1997, A\&A, 319, 1007

Varnière, P., Bjorkman, J. E., Frank, A., et al. 2006, ApJ, 637, L125

Watson, A. M., Stapelfeldt, K. R., Wood, K., \& Ménard, F. 2007, in: B. Reipurth, D. Jewitt \& K. Keil (eds.), Protostars and Planets $V$ (Tucson: University of Arizona Press), p. 523

Weidenschilling, S. 1977, MNRAS, 180, 57

Wolf, S. \& D'Angelo, G. 2005, ApJ, 619, 1114 